Notre Dame Journal of Formal Logic Volume XIX, Number 3, July 1978 NDJFAM

\title{
A NEW AXIOMATIZATION OF THE MIXED ASSOCIATIVE NEWMAN ALGEBRAS
}

\section{BOLESŁAW SOBOCIŃSKI}

In [1] M. H. A. Newman constracted, formalized and investigated two relatively complemented algebraic systems which he called "mixed nonassociative algebras" and "mixed associative algebras." In [2] $]^{1}$ and in the present paper only the latter system is investigated and it is called "mixed associative Newman algebras."' In [2] I have proved that this system can be axiomatized equationally in the following way:

(C) Any algebraic system

$$
\mathfrak{B}=\langle B,=,+, \times, \div\rangle
$$

with one binary relation $=$ and three binary operations,$+ \times$, and $\div$, is a relatively complemented mixed associative Newman algebra if, and only if, it satisfies the following postulates:

(i) The closure postulates:

$P 1 \quad[\exists a] . a \in B$

P2 $[a]: a \in B$.ว. $a=a$

1. An acquaintance with the paper [2] is presupposed. In the deductions presented in this paper the postulates $P 1-P 11$ and $R 1-R 10$ will be used mostly tacitly. An enumeration of the algebraic tables, $c f$. section 2.2 below, is a continuation of the enumeration of such tables given in [2], pp. 421-422, section 5. It should be noticed that in [2], p. 145, lines 7-9, the proof line 11 which appears there must be substituted by:

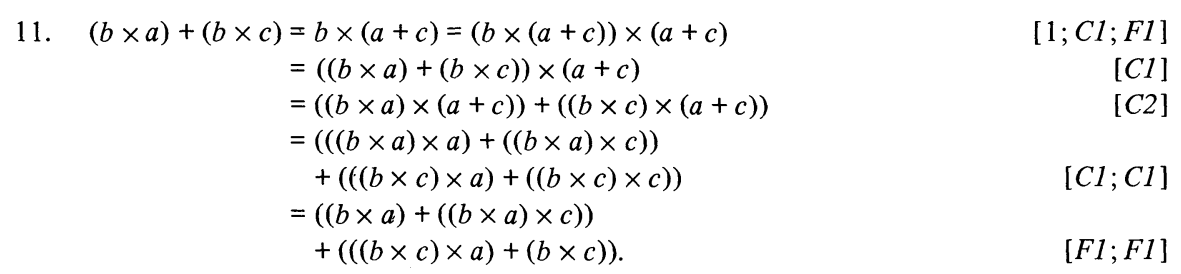

See Notre Dame Journal of Formal Logic, vol. XIX (1978), p. 192, Errata. 


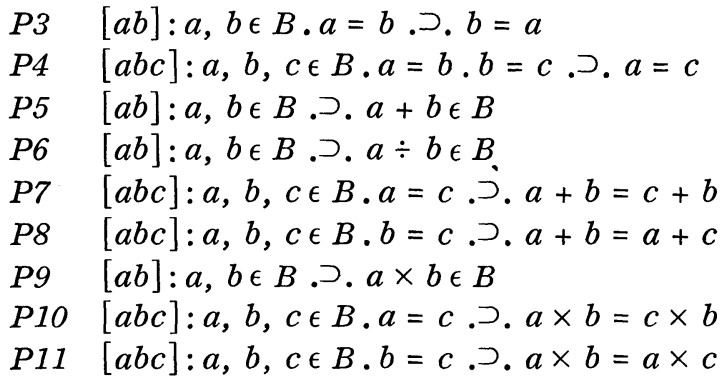

(ii) The mutually independent algebraic postulates:

$\begin{array}{ll}\text { G1 } & {[a b c]: a, b, c \in B . \supset . a \times(b+c)=(a \times b)+(a \times c)} \\ \text { G2 } & {[a b]: a, b \in B . \supset . a \times b=b \times a} \\ \text { G3 } & {[a b]: a, b \in B . \supset .(a \div b)+(b \times a)=a} \\ \text { G4 } & {[a b c]: a, b, c \in B . \supset .(a \div b) \times(b \times a)=c \div c^{2}}\end{array}$

Remark: Although the binary operation $\div$ is a primitive notion in the system $\mathfrak{B}$, the formulas concerning the extensionality of the relation $=$ with respect to this operation are not accepted axiomatically in $\mathfrak{B}$, since they are provable in this system, $c f$. the theses G22 and G24 in section 1 below.

Only some time after [2] was published I observed casually that in the system $\mathfrak{B}$ its primitive operations are not mutually independent. Namely, in the field of the postulate-system of $\mathfrak{B}$ we are able to define $\times$ by the operation $\div$ alone. In section 1 of this note I shall justify this fact and prove some theses which will be needed later. In section 2 an equational axiomatization of the mixed associative Newman algebras based only on two primitive operations + and $\div$ will be presented.

1 Let us assume the axioms $P 1-P 11$ and $G 1-G 4$. Then:

1.1 We have at our disposal the theses $65-G 24$ which are already proven in [2], pp. 419-420. Namely:

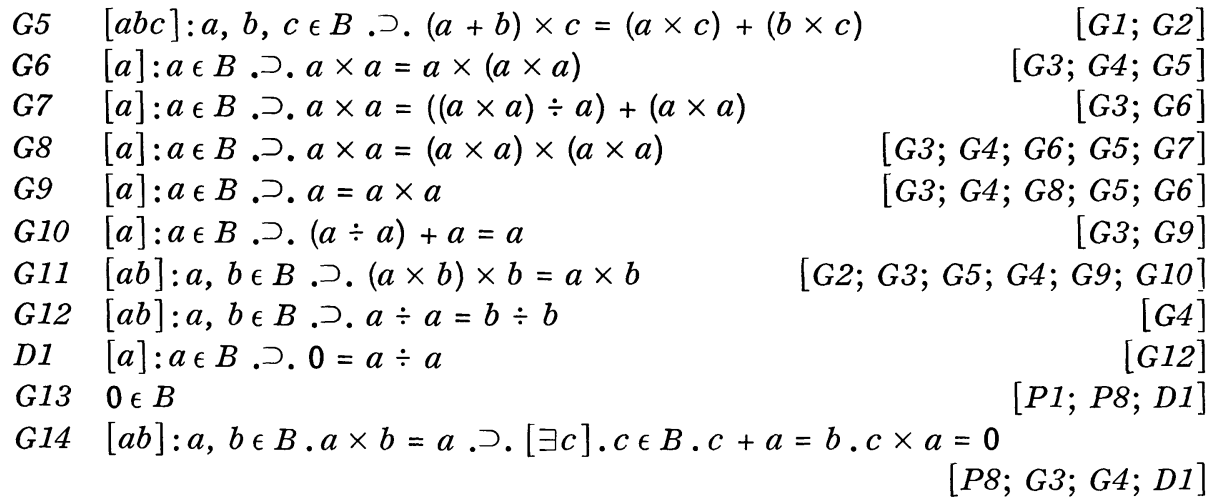

2. The mutual independence of the axioms $G 1, G 2, G 3$ and $G 4$ is established in [2], pp. 421-422, section 5 . 
G15 $[a b]: a, b \in B . b \times a=a . \supset .[\exists c] . c \in B . c+a=b . c \times a=0$

G16 $[a]: a \in B$.D. $0+a=a$

$[G 14 ; G 2]$

$[G 10 ; D 1]$

G17 $[a b c]: a, b, c \in B . \supset .(b \div b) \times a=c \div c$

$[G 12 ; G 9 ; G 4]$

G18 $[a]: a \in B$.ग. $a=a+0$

$[G 3 ; G 17 ; G 4 ; G 1 ; G 9 ; G 5 ; D 1]$

G19 $[a b]: a, b \in B$. . $(b \div a) \times b=b \div a$

$[G 3 ; G 1 ; G 9 ; G 4 ; D 1 ; G 18]$

G20 $[a b x]: a, b, x \in b . x+(a \times b)=b, x \times(a \times b)=0 . \supset . b+a=x$

$[G 19 ; G 2 ; G 5 ; G 2 ; G 4 ; D 1 ; G 1 ; G 3 ; G 9 ; G 19]$

G21 $[a b x] \therefore a, b, x \in B . \supset: b \div a=x . \equiv . x+(a \times b)=b . x \times(a \times b)=0$

$[G 3 ; G 4 ; D 1 ; G 20]$

G22 $[a b c]: a, b, c \in B . b=c . \supset . b \div a=c \div a$

$[P 11 ; G 3 ; G 4 ; P 8 ; G 20]$

G23 $[a b c d]: a, b, c, d \in B . c+a=b . c \times a=0 . d+a=b . d \times a=0 . \supset . c=d$

$[G 1 ; G 2 ; G 9 ; G 16 ; G 20]$

G24 $[a b c]: a, b, c \in B . a=c . \supset . b \div a=b \div c$

$[P 10 ; G 3 ; G 4 ; D 1 ; G 23]$

Thus, the extensional formulas G22 and G24, see Remark above, are the consequences of the postulate-system of $\boldsymbol{B}$.

1.2 Now, we proceed as follows:

G25 $[a b]: a, b \in B . \supset .(b \div a) \times(a \times b)=0$

G26 $[a b x]: a, b, x \in B .(a \times b)+x=b .(a \times b) \times x=0 . \supset . b \div a=x$

$[G 4 ; D 1]$

PR $[a b x]: \operatorname{Hp}(3) . \supset$.

$$
\begin{array}{rlr}
b \div a=(b \div a) \times b & =(b \div a) \times((a \times b)+x) & {[1 ; G 19 ; 2]} \\
& =((b \div a) \times(a \times b))+((b \div a) \times x) & {[G 1]} \\
& =0+((b \div a) \times x)=(b \div a) \times x & {[G 25 ; G 16]} \\
& =((b \div a) \times x)+0=((b \div a) \times x)+((a \times b) \times x) \\
& =((b \div a)+(a \times b)) \times x=b \times x & {[G 18 ; 3]} \\
& =((a \times b)+x) \times x=((a \times b) \times x)+(x \times x) & {[G 5 ; G 3]} \\
& =0+x=x & {[2 ; G 5]}
\end{array}
$$

G27 [ab]:a, $b \in B$. . $a \times b=a \div(a \div b)$

PR $[a b]: \operatorname{Hp}(1) . \supset$.

2. $((a \div b) \times a)+(a \times b)=(a \div b)+(b \times a)=a$.

$[1 ; G 19 ; G 2 ; G 3]$

3. $((a \div b) \times a) \times(a \times b)=(a \div b) \times(b \times a)=0$.

$[1 ; G 19 ; G 2 ; G 25]$

$a \times b=a \div(a \div b)$

$[1 ; G 26 ; 2 ; 3]$

Thesis G27 shows that in the system $\boldsymbol{B}$ the operations,$+ \times$, and $\div$ are not mutually independent.

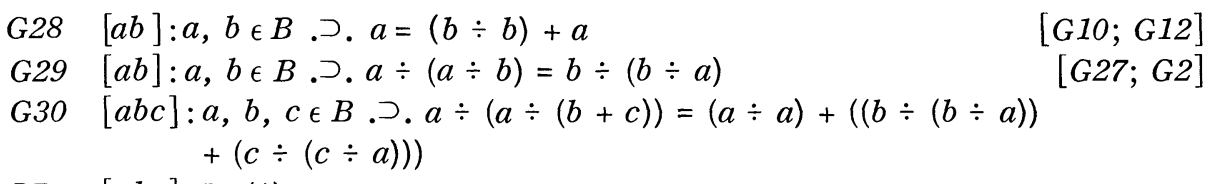

PR $\quad[a b c]: \operatorname{Hp}(1) . \supset$.

$a \div(a \div(b+c))=a \times(b+c)=(b+c) \times a$

$=(b \times a)+(c \times a)=(b \div(b \div a))+(c \div(c \div a))$

$[1 ; G 27 ; G 2]$

$=(a \div a)+((b \div(b \div a))+(c \div(c \div a)))$

$[G 5 ; G 27]$

G31 $[a b]: a, b \in B$.ว. $(a \div b)+(b \div(b \div a))=a$

[G28]

$[G 3 ; G 27]$

G32 $[a b c]: a, b, c \in B$.つ. $(a \div b) \div((a \div b) \div(b \div(b \div a)))=c \div c$

$[G 27 ; G 4]$ 
G33 $[a b x]: a, b, x \in B .(a \div b)+x=a .(a \div b) \div((a \div b) \div x)=a \div a$.

$$
\text { ว. } a \times b=x
$$

PR $[a b x]: \operatorname{Hp}(3)$.ว.

4. $(a \div b) \times x=a \div a$.

$a \times b=b \times a=(b \times a) \times a=(b \times a) \times((a \div b)+x)$

$[1 ; G 2 ; G 11 ; 2]$

$=((b \times a) \times(a \div b))+((b \times a) \times x)$

$=((a \div b) \times(b \times a))+((b \times a) \times x)$

$=(a \div a)+((b \times a) \times x)$

$=((a \div b) \times x)+((b \times a) \times x)$

$=((a \div b)+(b \times a)) \times x=a \times x$

$=((a \div b)+x) \times x=((a \div b) \times x)+(x \times x)$

$=(a \div a)+x=x$

$[4 ; G 9 ; G 28]$

G34 $[a b x] \therefore a, b, x \in B . a \times b=x . \supset:(a \div b)+x=a .(a \div b) \div((a \div b) \div x)=$ $a \div a$

PR $\quad[a b x] \therefore \mathrm{Hp}(2) . \supset$ :

3. $b \div(b \div a)=b \times a=a \times b=x$ :

$(a \div b)+x=a .(a \div b) \div((a \div b) \div x)=a \div a$

G35 $[a b x]:: a, b, x \in B . \supset \therefore a \times b=x . \equiv:(a \div b)+x=a$.

$$
(a \div b) \div((a \div b) \div x)=a \div a
$$

[G33; G34]

2 In this section we shall prove the valitity of the following formalization of mixed associative Newman algebras:

(D) Any algebraic system

$$
\boldsymbol{c}=\langle C,=,+, \div\rangle
$$

with one binary relation $=$ and two binary operations + and $\div$, is a relatively complemented mixed associative Newman algebra if, and only if, it satisfies the following postulates:

(i) The closure postulates:

$R 1 \quad[\exists a] . a \in C$

R2 $[a]: a \in C . \supset . a=a$

$R 3 \quad[a b]: a, b \in C . a=b . \supset . b=a$

$R 4 \quad[a b c]: a, b, c \in C . a=b . b=c . \supset . a=c$

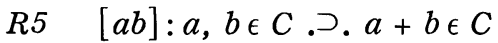

$R 6 \quad[a b]: a, b \in C . \supset . a \div b \in C$

$R 7 \quad[a b c]: a, b, c \in C . a=c . \supset . a \div b=c+b$

$R 8 \quad[a b c]: a, b, c \in C . b=c . \supset . a+b=a+c$

R9 [abc]: $a, b, c \in C . a=c$.Ј. $a \div b=c \div b$

$R 10[a b c]: a, b, c \in C . b=c . \supset . a \div b=a \div c$

and

(ii) The mutually independent algebraic postulates:

S1 $[a b c]: a, b, c \in C . \supset . a \div(a \div(b+c))=(a \div a)+((b \div(b \div a))$

$$
+(c \div(c \div a)))
$$

S2 $[a b]: a, b \in C . \supset .(a \div b)+(b \div(b \div a))=a$

S3 $[a b c]: a, b, c \in C . \supset .(a \div b) \div((a \div b) \div(b \div(b \div a)))=c \div c$ 


\section{Proof:}

2.1 Let us assume the postulates of the system $(\boldsymbol{C}$. Then:

S4 $[a b]: a, b \in C . \supset . a \div a=b \div b$

S5 $\quad[a b c d]: a, b, c, d \in C . \supset . a \div(a \div(b+c))=(d \div d)$

$$
+((b \div(b \div a))+(c \div(c \div a)))
$$

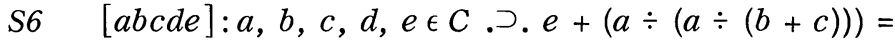

$[S 3, c / a ; S 3, c / b]$

$[S 1 ; S 4, b / d]$ $e+((d \div d)+((b \div(b \div a))+(c \div(c \div a))))$

$[R 8, a / e, b / a \div(a \div(b+c)), c /(d \div d)+((b \div(b \div c))+(c \div(c \div a))) ; S 5]$

S7 $\quad[a]: a \in C . \supset . a \div a=(a \div a)+(a \div a)$

PR $[a]: \operatorname{Hp}(1) . \supset$.

$a \div a=((a \div a) \div(a \div a))+((a \div a) \div((a \div a) \div(a \div a)))$

$$
\begin{aligned}
& =(a \div a)+((a \div a) \div(a \div a)) \\
& =(a \div a)+(a \div a)
\end{aligned}
$$

$[1 ; S 2, a / a \div a, b / a \div a]$

$[S 4, b / a \div a]$

$[S 4, b / a \div a]$

S8 $[a]: a \in C . \supset .(a \div(a \div a)) \div((a \div(a \div a)) \div(a \div a))=a \div a$

PR $[a]: \operatorname{Hp}(1) . \supset$.

$(a \div(a \div a)) \div((a \div(a \div a)) \div(a \div a))$

$=(a \div(a \div a)) \div((a \div(a \div a)) \div((a \div a)+(a \div a)))$

$=(a \div a)+(((a \div a) \div((a \div a) \div(a \div(a \div a))))+((a \div a)$

$\div((a \div a) \div(a \div(a \div a))))) \quad[S 5, a / a \div(a \div a), b / a \div a, c / a \div a, d / a]$

$=(a \div a)+((a \div a)+(a \div a))$

$=(a \div a)+(a \div a)=a \div a$

$[S 3, b / a, c / a]$

$[S 7 ; S 7]$

S9 $\quad[a]: a \in C . \supset .(a \div a) \div((a \div a) \div a)=a \div a$

PR $[a]: \operatorname{Hp}(1) . \supset$.

$(a \div a) \div((a \div a) \div a)=(a \div a) \div((a \div a) \div((a \div a)+(a \div(a \div a))))$

$[1 ; S 2, b / a]$
$[(a \div a)+(((a \div a) \div((a \div a) \div(a \div a)))+((a \div(a \div a)) \div((a \div(a \div a))$

$=(a \div a)+(((a \div a) \div((a \div a) \div(a \div a)))+((a \div(a \div a)) \div((a \div(a \div a))$ $\div(a \div a))))$ $[S 5, a / a \div a, b / a \div a, c / a \div(a \div a), d / a]$

$=(a \div a)+(((a \div a) \div(a \div a))+(a \div a))$

$=(a \div a)+((a \div a)+(a \div a))$

$[S 4, b / a \div a ; S 8]$

$=(a \div a)+(a \div a)=a \div a$

$[S 4, b / a \div a]$

$[s 7 ; s 7]$

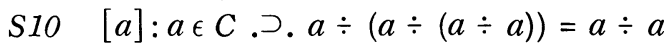

$[a]: \operatorname{Hp}(1) . \supset$.

$a \div(a \div(a \div a))=a \div(a \div((a \div a)+(a \div a)))$

$=(a \div a)+(((a \div a) \div((a \div a) \div a))+((a \div a) \div((a \div a) \div a)))$

$[S 5, b / a \div a, c / a \div a, d / a]$

$=(a \div a)+((a \div a)+(a \div a))=(a \div a)+(a \div a)=a \div a$

$[s 9 ; s 7 ; s 7]$

S11 [ab]:a, $b \in C$.ว. $a=(b \div b)+a$

PR $[a b]: \mathrm{Hp}(1)$.つ.

$$
\begin{aligned}
a= & (a \div(a \div(a \div a)))+((a \div(a \div a)) \div(((a \div(a \div a)) \div a))) \\
= & {[1 ; S 2, b / a \div(a \div a)] } \\
& (a \div a)+((a \div(a \div a)) \div((a \div(a \div a)) \div((a \div a)+(a \div(a \div a))))) \\
= & {[S 10 ; S 2, b / a] } \\
& (a \div a)+((a \div a)+(((a \div a) \div((a \div a) \div(a \div(a \div a))))+((a \div(a \div a)) \\
& \quad[(S 6, a / a \div(a \div a), b /(a \div a), a / a \div(a \div a), d / a, e / a \div a]
\end{aligned}
$$




$$
\begin{aligned}
& =(a \div a)+((a \div a)+((a \div a)+((a \div(a \div a)) \div((a \div(a \div a)) \\
& \div(a \div(a \div a)))))) \\
& =(a \div a)+((a \div a)+(((a \div(a \div a)) \div(a \div(a \div a)))+((a \div(a \div a)) \\
& \div((a \div(a \div a)) \div(a \div(a \div a)))))) \\
& {[S 4, b / a \div(a \div a)]} \\
& =(a \div a)+((a \div a)+(a \div(a \div a))) \quad[S 2, a / a \div(a \div a), b / a \div(a \div a)] \\
& =(b \div b)+a
\end{aligned}
$$

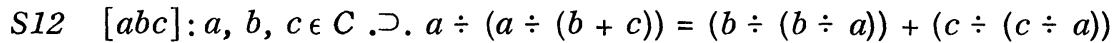

$$
[S 1 ; S 11, a /(b \div(b \div a))+(c \div(c \div a)), b / a]
$$

S13 [ab]:a, $b \in C . \supset . a \div(a \div b)=b \div(b \div a)$

PR $[a b]: \mathrm{Hp}(1) . \supset$.

$a \div(a \div b)=a \div(a \div((a \div a)+b))$

$=((a \div a) \div((a \div a) \div a))+(b \div(b \div a)$

$=(a \div a)+(b \div(b \div a))=b \div(b \div a)$

$[1, S 11, a / b, b / a]$

$[S 12, b / a \div a, c / b]$

$[S 9 ; S 11, a / b \div(b \div a), b / a]$

$S 14[a b] \therefore a, b \in C . \supset:[\exists x]: x \in C .(a \div b)+x=a .(a \div b) \div((a \div b)$

$\div x)=a \div a$

PR $[a b] \therefore \mathrm{Hp}(1) . \supset$ :

2. $b \div(b \div a) \in C: \quad[1 ; R 6, a / b, b / a ; R 6, a / b, b / b \div a]$

$[\exists c]: c \in C .(a \div b)+x=a .(a \div b) \div((a \div b) \div x)=a \div a$

S15 $[a b x y]: a, b, x, y \in C .(a \div b)+x=a .(a \div b) \div((a \div b) \div x)$

$[1 ; 2 ; S 2 ; S 3, c / a]$

$=a \div a \cdot(a \div b)+y=a .(a \div b) \div((a \div b) \div y)=a \div a . \supset . x=y$

PR $[a b x y]: \operatorname{Hp}(5) . \supset$.

6. $x \div(x \div a)=x \div(x \div(((a \div b)+y))$

$=((a \div b) \div((a \div b) \div x))+(y \div(y \div x))$

$=(a \div a)+(y \div(y \div x))$

$=((a \div b) \div((a \div b) \div y))+(x \div(x \div y))$

$=y \div(y \div((a \div b)+x))=y \div(y \div a)$.

7. $x \div(x \div a)=x \div((x \div((a \div b)+x))$

$=((a \div b) \div((a \div b) \div x))+(x \div(x \div x))$

$[S 12, a / x, b / a \div b, c / y]$

$[5 ; S 13, a / y, b / x]$

$[S 12, a / y, b / a \div b, c / x ; 2]$

$=(a \div a)+(x \div(x \div x))$

$[S 12, a / x, b / a \div b, c / x]$

$=(x \div x)+(x \div(x \div x))=x$.

8. $x \div(x \div a)=y \div(y \div a)=y \div(y \div((a \div b)+y))$

$=((a \div b) \div((a \div b) \div y))+(y \div(y \div y))$

$[S 4, b / x ; s 2, a / x, b / x]$

$=(a \div a)+(y \div(y \div y))$

$[1 ; 6 ; 4]$

$=(y \div y)+(y \div(y \div y))=y$.

$[S 12, a / y, b / a \div b, c / y]$

$x=y$

$[S 4, b / y ; S 2, a / y, b / y]$

$[7 ; 8]$

Since we have $S 14$ and $S 15$, we can introduce into the system the following definition:

$D 1 \quad[a b x] \therefore a, b, x \in C . \supset: a \times b=x . \equiv .(a \div b)+x=a .(a \div b)$

$\div((a \div b) \div x)=a \div a$

$[S 14 ; S 15]$

S16 [ab]:a, $b \in C . \supset . a \times b=a \div(a \div b)$

PR $[a b]: \operatorname{Hp}(1)$.つ.

$a \times b=b \div(b \div a)=a \div(a \div b) \quad[1 ; D 1, x / b \div(b \div a) ; S 2 ; S 3, c / a ; S 13]$

S17 $[a b c]: a, b, c \in C . \supset a \times(b+c)=(a \times b)+(a \times c)$

PR $[a b c]: \operatorname{Hp}(1) . \supset$. 


$$
\begin{aligned}
& a \times(b+c)=a \div(a \div(b+c)) \\
& =(b \div(b \div a))+(c \div(c \div a)) \\
& =(a \div(a \div b))+(a \div(a \div c)) \\
& =(a \times b)+(a \times c) \\
& {[a b]: a, b \in C . \supset .(a \times b)=(b \times a)}
\end{aligned}
$$$$
S 19[a b]: a, b \in C . \supset .(a \div b)+(b \times a)=a
$$$$
\text { S20 }[a b c]: a, b, c \in C \text {.Ј. }(a \div b) \times(b \times a)=c \div c
$$

PR $\quad[a b c]: \mathrm{Hp}(1) . \supset$.

$$
(a \div b) \times(b \times a)=(a \div b)+((a \div b)+(b \div(b+a)))
$$

$[S 16, a / a \div b, b / b \times a ; S 16, a / b, b / a]$

$$
=c \div c
$$

$S 21[a b]: a, b \in C . \supset . a \times b \in C$

S22 [abc]: $a, b, c \in C . a=c . \supset a \times b=c \times b$

PR $[a b c]: \mathrm{Hp}(2) . \supset$.

$$
a \times b=a \div(a \div b)=c \div(c \div b)=c \times b
$$

S23 $[a b c]: a, b, c \in C . b=c . \supset . a \times b=a \times c$

$[1 ; S 16, b / b+c]$

$[S 13 ; S 13, b / c]$

$[S 16 ; S 16, b / c]$

$[S 16 ; S 13 ; S 16, a / b, b / a]$ $[S 16, a / b, b / a ; S 2]$

PR $[a b c]: \operatorname{Hp}(2) . \supset$.

$a \times b=b \times a=c \times a=a \times c \quad[1 ; S 18 ; S 22, a / b, b / a, S 18, a / c, b / a]$

2.2 The mutual independence of the axioms $S 1, S 2$, and $S 3$ is established by using the following algebraic tables (matrices):

\begin{tabular}{cc|cccc} 
& \multirow{4}{*}{$\mathfrak{M} 13$} & $O$ & $\alpha$ & $\beta$ & $\gamma$ \\
\hline & $O$ & $O$ & $\alpha$ & $\beta$ & $\gamma$ \\
& $\alpha$ & $\alpha$ & $\alpha$ & $\beta$ & $\gamma$ \\
& $\beta$ & $\beta$ & $\beta$ & $\beta$ & $\alpha$ \\
& $\gamma$ & $\gamma$ & $\gamma$ & $\gamma$ & $\gamma$
\end{tabular}

$\mathfrak{M} 14$\begin{tabular}{c|cc}
+ & $\alpha$ & $\beta$ \\
\hline$\alpha$ & $\alpha$ & $\beta$ \\
$\beta$ & $\beta$ & $\alpha$
\end{tabular}

\begin{tabular}{ll|ll}
$\mathfrak{M} 15$ & + & $\alpha$ & $\beta$ \\
\hline & $\alpha$ & $\alpha$ & $\alpha$ \\
$\beta$ & $\beta$ & $\beta$
\end{tabular}

\begin{tabular}{l|llll}
$\doteqdot$ & $O$ & $\alpha$ & $\beta$ & $\gamma$ \\
\hline$O$ & $O$ & $O$ & $O$ & $O$ \\
$\alpha$ & $\alpha$ & $O$ & $\alpha$ & $\alpha$ \\
$\beta$ & $\beta$ & $\beta$ & $O$ & $\beta$ \\
$\gamma$ & $\gamma$ & $\gamma$ & $\gamma$ & $O$
\end{tabular}

\begin{tabular}{l|ll}
$\div$ & $\alpha$ & $\beta$ \\
\hline$\alpha$ & $\alpha$ & $\alpha$ \\
$\beta$ & $\alpha$ & $\alpha$
\end{tabular}

\begin{tabular}{l|ll}
$\div$ & $\alpha$ & $\beta$ \\
\hline$\alpha$ & $\alpha$ & $\alpha$ \\
$\beta$ & $\beta$ & $\beta$
\end{tabular}

Concerning the matrix $\mathfrak{M 1 4} c f . \mathfrak{M l l}$ in [2], p. 422. Since:

(a) matrix $\mathfrak{M} 13$ verifies $S 2$ and $S 3$, but falsifies $S 1$ for $a / \alpha, b / \beta$, and $c / \gamma$ : (i) $\alpha \div(\alpha \div(\beta+\gamma))=\alpha \div(\alpha \div \alpha)=\alpha \div O=\alpha$, and (ii) $(\alpha \div \alpha)+((\beta \div(\beta \div \alpha))+$ $(\gamma \div(\gamma \div \alpha)))=O+((\beta \div \beta)+(\gamma \div \gamma))=O+(O+O)=O+O=O$,

(b) matrix $\mathfrak{M 1 4}$ verifies $S 1$ and $S 3$, but falsifies $S 2$ for $a / \beta$ and $b / \beta$ : (i) $(\beta \div$ $\beta)+(\beta \div(\beta \div \beta))=\alpha+(\beta \div \alpha)=\alpha+\alpha=\alpha$, and (ii) $\beta=\beta$,

(c) matrix $\mathbf{M 1 5}$ verifies $S 1$ and $S 2$, but falsifies $S 3$ for $a / \alpha, b / \alpha$, and $c / \beta$ : (i) $(\alpha \div \alpha) \div((\alpha \div \alpha) \div(\alpha \div(\alpha \div \alpha)))=\alpha \div(\alpha \div(\alpha \div \alpha))=\alpha \div(\alpha \div \alpha)=\alpha \div \alpha=\alpha$, and (ii) $\beta \div \beta=\beta$,

we know that the axioms $S 1, S 2$, and $S 3$ are mutually independent. 
2.3 An inspection of the deductions presented in sections 1 and 2.1 shows that

(i) The theses $P 1, P 2, P 3, P 4, P 5, P 6, P 7, P 8, P 9, P 10, P 11, G 1, G 2, G 3$, G4, G22, G24, G30, G31, and $G 32$ of $\mathfrak{B}$ correspond synonymously and respectively to the theses $R 1, R 2, R 3, R 4, R 5, R 6, R 7, R 8, S 21, S 22, S 23$, $S 17, S 18, S 19, S 20, R 9, R 10, S 1, S 2$, and $S 3$ of $\mathbf{C}$;

and, moreover, that

(ii) Definition $D 1$ which is introduced into the system (c) corresponds synonymously to the thesis G35 which is provable in the field of the system $\mathfrak{B}$.

Therefore, it follows from the points (i) and (ii) that the systems $\mathfrak{B}$ and (c) are inferentially equivalent up to isomorphism. Thus, the proof that the system $\boldsymbol{C}$ is a correct axiomatization of the mixed associative Newman algebras is complete.

\section{REFERENCES}

[1] Newman, M. H. A., "Relatively complemented algebras," The Journal of the London Mathematical Society, vol. 17 (1942), pp. 32-47.

[2] Sobociński, B., “An equational axiomatization and a semi-lattice characterization of mixed associative Newman algebras," Notre Dame Journal of Formal Logic, vol. XIII (1972), pp. 407-423.

University of Notre Dame

Notre Dame, Indiana 